\title{
SPECTRAL ANALYSIS OF FOUR RECYCLED PULSARS
}

\author{
R. S. Foster, L. Fairhead, And D. C. BaCker \\ Astronomy Department and Radio Astronomy Laboratory, University of California at Berkeley
}

\begin{abstract}
Flux density measurements of four recycled pulsars, PSR 1620-26, PSR 1821-24, PSR $1855+09$, and PSR 1.937+21, have been made to determine their spectral indices in the range between $425 \mathrm{MHz}$ to $3 \mathrm{GHz}$. The four objects are shown to have indices that range from -1.3 to -2.6 . The luminosities of these four pulsars are spread over nearly three orders of magnitude. An analytic pulse component model is developed for each object. Individual components are allowed to have different spectral indices and hence different component ratios as a function of frequency. Component separations are evaluated as a function of frequency. The analytic models are used to determine dispersion measures with a precision better than $0.01 \mathrm{pc} \mathrm{cm}^{-3}$ for each object. The intrinsic pulse widths of each of these objects is less than $\sim 4$ percent of their respective pulse periods.
\end{abstract}

\section{Observations}

The observational data were collected as part of a continuing pulsar timing experiment using the 43$m$ radio telescope at the National Radio Astronomy Observatory (NRAO) in Green Bank, West Virginia (Foster and Backer 1990). Observations were conducted over 16 days between 1989 August 8 and 1989 August 27 at frequencies between $425 \mathrm{MHz}$ and $3 \mathrm{GHz}$. Supplemental observations were obtained at 800 and $1330 \mathrm{MHz}$ during four days in both 1989 October and 1990 January. Data for this experiment were collected using the NRAO spectral processor in its pulsar timing configuration.

The spectral processor is capable of sampling a $20-\mathrm{MHz}$ wide, single side band, filtered baseband voltage signal at $\sim 40 \mathrm{MHz}$ and performing 512-point Fast Fourier Transforms (FFT) every $\sim 12.8 \mu \mathrm{s}$ on each of two polarization channels. The typical time resolution across the pulse period for the pulsars studied in this paper varies from $\sim 12.8 \mu$ s to $\sim 128 \mu$ s depending on the optimal configuration for the spectral processor hardware for the various pulsars and observing frequencies. We varied the total observing bandwidth from $20 \mathrm{MHz}$ down to $2.5 \mathrm{MHz}$ depending on the observing frequency and dispersion measure of the particular pulsar. The intensity data are accumulated in a two dimensional frequency and time (phase) array for the length of the integration. Dedispersion is done in software as a post processing procedure after filtering the bandpass for interference. Calibration measurements were made against the extragalactic continuum sources $3 \mathrm{C} 48$ and $3 \mathrm{C} 286$ at least once per night at each frequency. All the data are time tagged with the time of a local quartz clock phase locked to within $\sim 100$ ns with the local NRAO hydrogen maser, which in turn is monitored daily against UTC as kept by the U. S. Naval Observatory and transferred by the Global Positioning System of satellites.

\section{Analysis}

The spectra of individual components from each of the four pulsars observed in this study are presented in figure 1 . The values of the continuum spectral indices are given in table 1 , where $S(\nu)=S_{0} \nu^{\alpha}, S_{0}$ is the mean flux density at the reference frequency of $1 \mathrm{GHz}$ and $\alpha$ is the spectral index. The four objects have total flux density spectral indices that range from a low value of -1.3 up to -2.6 . Individual pulse component ratios vary as a function of frequency and hence the spectral indices of the individual components are different from the mean spectral index of the pulsar. We devised a pulse component model for each of the four objects based on the multifrequency data collected in $1989 \mathrm{Au}$ gust. The model includes pulse component width(s) (Gaussian) as a function of frequency, component separation(s), and the spectral index of each component. The spectral indices of the individual components, their absolute flux densities, and the component widths are given. We reference the models to a fictitious $1-\mathrm{GHz}$ profile. A fit assuming constant pulse width as a function of frequency and a fit assuming a variable pulse width as a function of frequency are given. The analytic models are used to determine arrival times which are in turn used to solve for a dispersion measure toward each pulsar. The component model $P(t)$ is the sum of $k$ Gaussian components in the profile. The model used to generate the analytic profile $I(t)$ is given as

$$
I\left(t_{i}\right)=\mathrm{III}(\Delta t) \cdot\left[P \star h_{\mathrm{DM}} \star h_{\mathrm{ISS}}\right], i=[1, N]
$$



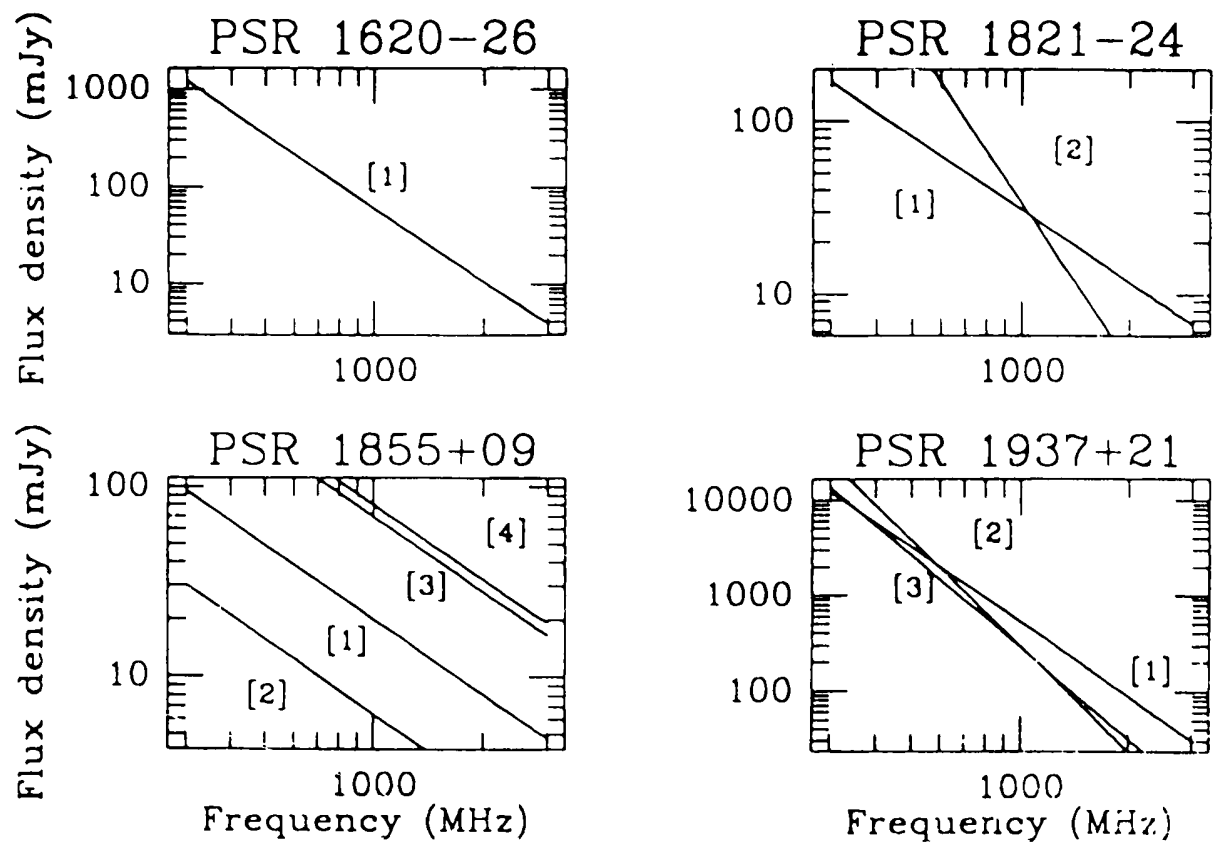

Figure 1 The spectra of individual components from each of the four pulsars observed.

where the analytic profile $P(t)$ has been convolved by two instrumental functions $h_{\mathrm{DM}}(t)$ and $h_{\mathrm{ISS}}(t)$, and sampled uniformly over $N$ bins. The uniform sampling time $\Delta t$ is represented by the dot product of the convolved profile with the Shah function, $\operatorname{III}(\Delta t)$.

Dispersion sweep of the pulsed signal through a filter channel creates a temporal impulse response $h_{\mathrm{DM}}(t)$ which is related to the bandpass shape

$$
h_{\mathrm{DM}}(t)=\operatorname{sinc}^{2}\left(\pi t / \tau_{\mathrm{DM}}\right) \text {, }
$$

where

$$
\tau_{\mathrm{DM}}=8.3 \mu \mathrm{s} \Delta \nu(\mathrm{MHz}) \mathrm{DM}\left(\mathrm{pccm}^{-3}\right) \nu^{-3}(\mathrm{GHz}) \text {. }
$$

The smearing from interstellar multipath propagation can be derived from observations or can be estimated as

$$
h_{\mathrm{ISS}}(t)=e^{-t / \text { rSs }}
$$

where

$\tau_{\mathrm{SS}}=0.61 \mu \mathrm{s}\left(\frac{D}{5 \mathrm{kpc}}\right)\left(\frac{\nu}{1 \mathrm{GHz}}\right)^{-4.4}\left(\frac{D M}{71 \mathrm{pc} \mathrm{cm}^{-3}}\right)^{0.5}$

which is normalized to parameters for PSR $1937+21$ (Cordes et al. 1990).

An iterative regression routine was used to deconvolve the known response functions from the unknown Gaussian profiles. The results from the pulse modeling are used to create analytic pulse profiles. The model templates are cross-correlated against the data to determine a relative arrival time. The actual arrival time is then computed from the delay of the template-data cross-correlation function, after correction for the delay associated with the peak of the strongest component at $1 \mathrm{GHz}$.

We computed the sharpness parameter $\Lambda=$ $1 / \int_{0}^{P}\left|u^{\prime}(t)\right|^{2} d t$ (Downs and Reichley 1983), where $u^{\prime}(t)$ is the derivative of the normalized pulse profile $u(t)$. The timing factor $T F=\sqrt{\Lambda P} / S_{\text {peak }}$ gives the relative timing ability of each pulsar. To compute the actual arrival time precision for a given telescope divide $T F$ by the square root of the integration time and bandwidth and multiply by the system temperature and gain factor of the telescope. The sharpness and $T F$ parameter from the NRAO data are given in table 1 .

\section{Individual objects}

A summary of the results for each pulsar are given below and the component separations are given in table 2.

\section{PSR 1620-26}

The pulse profile of this object is dominated by a single component with an inferred width of $\lesssim 400 \mu \mathrm{s}(\mathrm{FWHM})$ at $1 \mathrm{GHz}$. The mean flux density at $1 \mathrm{GHz}$ is $3.3 \pm 1.0 \mathrm{mJy}$ with a steep spectral index of $-2.5 \pm 0.2$. Of the four objects in our study only PSR $1937+21$ has a steeper spectral index. An interesting feature of the PSR 1620-26 profile is that it appears to have a "shoulder" component on each side of the main component. A typical profile obtained in several hours of integration at $1330 \mathrm{MHz}$ is displayed in figure 2 . 
Table 1 Measured and inferred values

\begin{tabular}{|c|c|c|c|c|}
\hline Parameter & PSR 1620-26 & PSR 1821-24 & PSR 1855+09 & PSR 1937+21 \\
\hline Flux density (mJy) & $3.3 \pm 0.3$ & $3.9 \pm 0.4$ & $10.7 \pm 1.1$ & $25.9 \pm 2.6$ \\
\hline Index & $-2.5 \pm 0.2$ & $-2.3 \pm 0.2$ & $-1.3 \pm 0.1$ & $-2.6 \pm 0.1$ \\
\hline Distance (kpc) & $2.1^{a}$ & $5.8^{a}$ & $0.5^{b}$ & $5.0^{c}$ \\
\hline \multicolumn{5}{|l|}{ Luminosity (mJy $\mathrm{kpc}^{2}$ ) } \\
\hline at $400 \mathrm{MHz}$ & 140 & 1100 & 9 & 7000 \\
\hline$C_{\mathrm{N}}^{2}\left(10^{-3} m^{-20 / 3}\right)$ & 1.0 & 19 & 2.2 & 0.3 \\
\hline$\tau_{\text {DISS }}(\mathrm{s})$ & 43 & 6 & 1800 & 310 \\
\hline$\tau_{\text {RISS }}($ days $)$ & 1.4 & 63 & 6.6 & 15 \\
\hline $\mathrm{DM}(\mathrm{pccm}-3)$ & $62.888 \pm 0.009$ & $119.8338 \pm 0.0016$ & $13.296 \pm 0.007$ & $71.03569 \pm 0.00011$ \\
\hline$\Lambda(\mu \mathrm{s})$ & 187 & 33 & 51 & 12 \\
\hline $\mathrm{TF}$ at $1 \mathrm{GHz}(\mu \mathrm{s} / \mathrm{mJy})$ & 24 & 9.3 & 6.5 & 0.25 \\
\hline \multicolumn{5}{|l|}{ Main pulse width } \\
\hline$(\mathrm{FWHM})$ at $1 \mathrm{GHz}(\mu \mathrm{s})$ & $400 \pm 40$ & $130 \pm 25$ & $223 \pm 31$ & $49 \pm 5$ \\
\hline
\end{tabular}

'Webbink (1985), ${ }^{b}$ Rawley, Taylor, and Davis (1988), ${ }^{\circ}$ Heiles et al. (1983).

Table 2 Component separation

\begin{tabular}{|crr|}
\hline Pulsar & $\begin{array}{r}\text { Separation } \\
(\mu s)\end{array}$ & (degrees) \\
\hline $\begin{array}{lrr}1821-24 \\
\text { (component [2] - component [1]) }\end{array}$ & $913 \pm 12$ & $107.6 \pm 1.4$ \\
1855+09 & & \\
(main pulse [3] - interpulse [2]) & $2146 \pm 105$ & $144.1 \pm 7.0$ \\
(main pulse [4] - main pulse [3]) & $283 \pm 24$ & $19.0 \pm 1.6$ \\
(interpulse [2] - interpulse [1]) & $406 \pm 197$ & $27.3 \pm 13.2$ \\
1937+21 & & \\
(main pulse [1] - inter pulse [3]) & $744.9 \pm 1.3$ & $172.1 \pm 0.3$ \\
(notch pulse [2] - main pulse [1]) & $37.0 \pm 3.3$ & $8.6 \pm 0.8$ \\
\hline
\end{tabular}

- Separations are specified by the algebraic difference between arrival times of components given in column 1 . No significant frequency dependence of the separations are found.

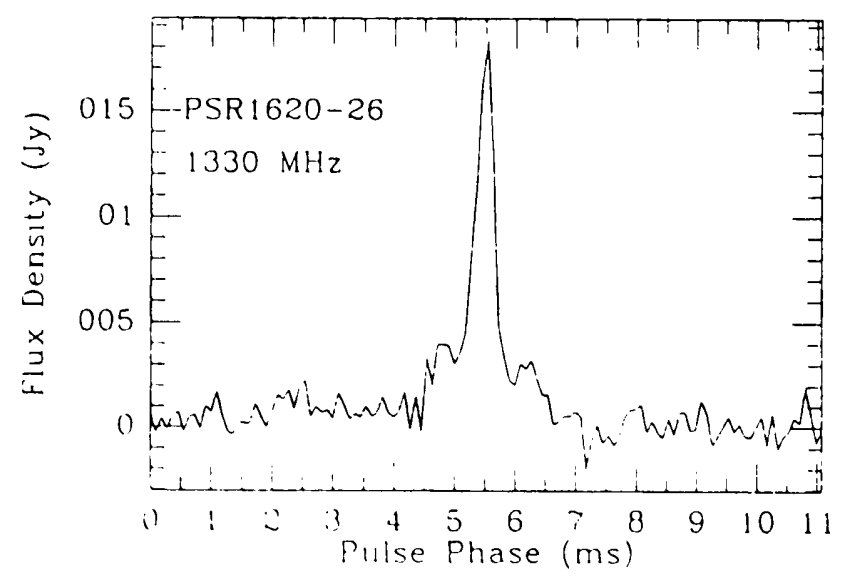

Figure 2 A typical profile of PSR 1620-26.

\section{PSR 1821-24}

The dominant feature of this pulsar is the neariy equal power emitted from two components separated by $116^{\circ}$. The separation between these components is nearly frequency independent in the range between 800 and $1705 \mathrm{MHz}$. The total flux density from this object has a spectral index of

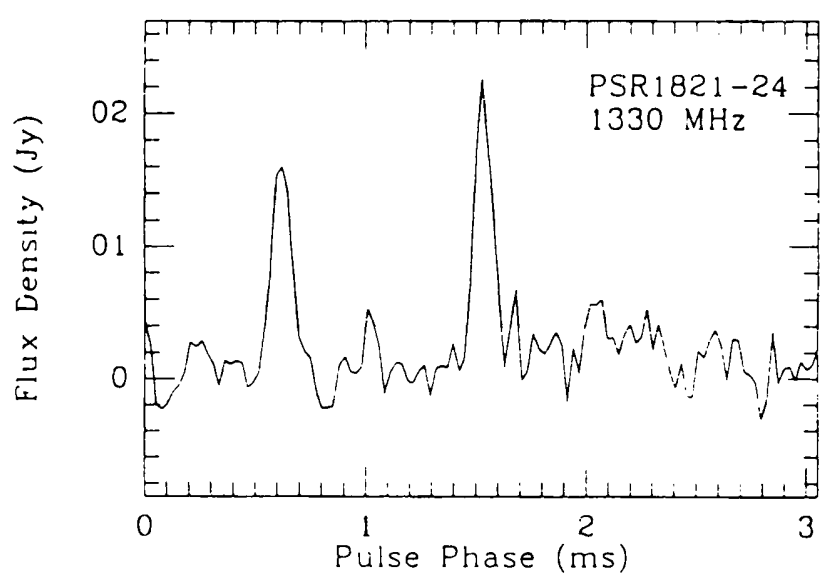

Figure 3 A profile of PSR 1821-24 obtained in several hours of integration at $1330 \mathrm{MHz}$.

$-2.3 \pm 0.2$ while the two components individually have spectral indices of $-1.4 \pm 0.2$ and $-3.1 \pm 0.2$. In fact, the strongest component changes between 800 and $1330 \mathrm{MHz}$. There is broad emission across one-half of the profile with comparable power to the total pulsed emission which gives a general "sinusoidal" characteristic to the baseline. This feature 
was also identified by Lyne et al. (1987) in the original discovery observations made at Jodrell Bank. The lower frequency observations indicate a strong dependence of pulse widths on frequency for this object. We conclude that the width increase is caused by broadening of the pulse width through multipath interstellar scattering. This pulse broadening leads us to infer a scintillation bandwidth of $\sim 1.4 \mathrm{kHz}$ at $1 \mathrm{GHz}$. Dispersion measure variations can be discerned for this object at a level of $0.02 \mathrm{pc} \mathrm{cm}^{-3}$ over six months of observations.

\section{PSR 1855+09}

Pulsar 1855+09 has the most complicated structure of the four pulsars observed in this study and the shallowest spectral index $(-1.3 \pm 0.2)$. The pulsar was successfully observed over the frequency range from $425 \mathrm{MHz}$ to $3000 \mathrm{MHz}$. The pulsar is dominated by the "main pulse" (our components [3] and [4]) and a preceding "interpulse" (our components [1] and [2]) which are separated by $144 .^{\circ} 1 \pm 7 .^{\circ} 0$. Each of these pulses in turn are the sum of two Gaussian components separated by $19 .^{\circ} 0 \pm 1 .^{\circ} 6$ and $27.3 \pm 13.2$, respectively, according to our data. Thorsett and Stinebring (1990) find evidence for two additional "shoulder" components, but we lack the sensitivity to detect these in our data. The flux density of PSR $1855+09$ varied widely during these observations, by a factor of four at $1665 \mathrm{MHz}$, due presumably to short time-scale diffractive scintillations.

\section{PSR 1937+21}

The data we collected on PSR $1937+21$ were supplemented by high resolution data obtained by Cordes et al. (1990) at the Arecibo Observatory at $430 \mathrm{MHz}$. The object PSR $1937+21$ has three components; the main pulse, an interpulse, and a "notch" pulse which follows the "main pulse." We are able to resolve the "notch" component in our data between $1330 \mathrm{MHz}$ and $3000 \mathrm{GHz}$ using a simple Gaussian component analysis after adding in the effect of dispersion smearing and interstellar scattering. The unweighted mean separation between the resolved main pulse and the interpulse is $744.9 \pm 1.3 \mu \mathrm{s}$. Dispersion measure variations at a level of $0.001 \mathrm{pc} \mathrm{cm}^{-3}$ are present over six months of observations.

\section{Conclusions}

The range of spectral indices seen in the individual pulse components from the four recycled pulsars indicate that there may be a continuous distribution of spectral indices ranging from $\alpha \sim-1$ to -3 . The three fastest known pulsars, PSR 1821-24, PSR 1937+21, and PSR 1957+20 (see Fruchter et al. 1990) all have at least one very steep $(\alpha \leq-3)$ component. Each of the four recycled pulsars examined in this paper have pulse widths at $1 \mathrm{GHz}$ that are around less than $4 \%$ of the pulse period. Except for PSR 1821-24, none of these objects show any strong frequency dependence on the pulse width.

Our observations indicate that the range of luminosities is nearly $10^{3}$ among these four recycled pulsars. Assuming a standard luminosity distribution model (e.g. Taylor and Manchester 1977) this implies that pulsars like PSR $1855+09$ will be $\sim 10^{4}$ times more numerous than pulsars like PSR 1937+21. Examining the two objects in our study that are found in globular clusters we notice that they differ by $\sim 10$ in luminosity. This implies that there should be at least 10 objects similar to PSR 1620-26 for every object similar to PSR 1821-24, if the cluster luminosity function matches that of field pulsars. There could be as many as 100 times more low luminosity objects similar to PSR 1855+09 than objects like the globular cluster pulsar PSR 1821-24.

If the luminosity function of globular cluster pulsars is similar to the luminosity function inferred for field pulsars we must conclude that a large population of low luminosity objects similar to PSR $1855+09$ exists in globular clusters. A substantial fraction of radio pulsars and possibly recycled radio pulsars may have "shallow" spectral emission ( $\alpha \gtrsim-1.5$ ). Radio surveys of continuum flux measurements of globular clusters should not exclude clusters with spectral indices even as low as $\alpha \sim-1$, since this may indicate the presence of "shallow" spectrum pulsar emission. Further investigation of the spectral emission properties of recycled pulsars will aid in determining if a "shallow" and "steep" spectrum classification of recycled pulsars is warranted.

\footnotetext{
Acknowledgments: The authors of this paper would like to express our thanks to $M$. Clark and J. R. Fisher for their work on the NRAO Green Bank spectral processor. The National Radio Astronomy Observatory is operated by Associated Universities, Inc., through support from the National Science Foundation. Our research was partially supported by the Center for Particle Astrophysics, a National Science Foundation Science and Technology Center operated by the University of California at Berkeley under Cooperative Agreement No. AST-8809616, and by grant AST-8719094 from the National Science Foundation. This paper summarizes a portion of a longer work by the same authors currently in preparation for submission to The Astrophysical Journal.
} 